\title{
Damping of self-generated Alfvén waves in a partially ionized medium and the grammage of cosmic rays in the proximity of supernova remnants
}

\author{
S. Recchia, ${ }^{a, b, *}$ D. Galli, ${ }^{c}$ L. Nava, ${ }^{d}$ M. Padovani, ${ }^{c}$ S. Gabici, ${ }^{e}$ A. Marcowith, ${ }^{f}$ \\ V. Ptuskin ${ }^{g}$ and G. Morlino ${ }^{c}$ \\ ${ }^{a}$ Department of Physics, University of Torino, via P. Giuria, 1, 10125 Torino, Italy \\ ${ }^{b}$ Istituto Nazionale di Fisica Nucleare, via P. Giuria, 1, 10125 Torino, Italy \\ ${ }^{c}$ INAF-Osservatorio Astrofisico di Arcetri, Largo E. Fermi 5, 50125 Firenze, Italy \\ dINAF-Osservatorio Astronomico di Brera, Via Bianchi 46, I-23807 Merate, Italy \\ ${ }^{e}$ Université de Paris, CNRS, Astroparticule et Cosmologie, F-75006 Paris, France \\ ${ }^{f}$ Laboratoire Univers et particules de Montpellier, Université Montpellier/CNRS, F-34095 Montpellier, \\ France \\ ${ }^{g}$ Pushkov Institute of Terrestrial Magnetism, Ionosphere and Radiowave Propagation, 108840, Troitsk, \\ Moscow, Russia \\ E-mail: sarah.recchia@unito.it
}

\begin{abstract}
We study the damping of Alfvén waves generated by the cosmic ray resonant streaming instability as due to ion-neutral damping, turbulent damping and non linear Landau damping, in the warm ionized and warm neutral phases of the interstellar medium. We do so in the context of the cosmic ray escape and propagation in the proximity of supernova remnants. For the ion-neutral damping, state-of-the-art damping coefficients are used, where the momentum transfer and charge exchange cross sections between various species of ions and neutrals are computed in detail or measured. We investigate whether the self-confinement of cosmic rays nearby sources can appreciably affect the grammage. In fact, if this is the case, the standard picture, in which CR secondaries are produced during the whole time spent by cosmic rays throughout the Galactic disk, should be deeply revisited. We show that the ion-neutral damping and the turbulent damping effectively limit the residence time of cosmic rays in the source proximity, so that the grammage accumulated near sources is found to be negligible, contrary to what was previously suggested.
\end{abstract}

$37^{\text {th }}$ International Cosmic Ray Conference (ICRC 2021)

July 12 th - 23rd, 2021

Online - Berlin, Germany

\footnotetext{
*Presenter
} 


\section{Introduction}

We examine the escape of cosmic rays (CRs) from supernova remnants (SNRs) and their propagation in the source region as due to the scattering on Alfvén waves generated by the CR resonant streaming instability. Such waves are produced at the resonant scale, namely at wavenumber $k \sim 1 / r_{\mathrm{L}}$, where $r_{\mathrm{L}}=\gamma m_{p} c^{2} / e B$ is the particle Larmor radius $\left(m_{p}\right.$ is the proton mass, $B$ is the magnetic field strength and $\gamma$ the Lorentz factor). The wave density is limited by several damping processes, in particular by $(i)$ ion-neutral damping in a partially ionized medium $[1,2]$, for which we adopt up-to-date damping coefficients, based on an accurate experimental or theoretical determination of the relevant cross sections; (ii) turbulent damping, due to the interaction of a wave with background counter-propagating Alfvén wave packets (that we indicate as FG, after [3]); (iii) non-linear Landau (NLL) damping, due to the interaction of background thermal ions with the beat of two interfering Alfvén waves (see e.g. [4]). The large residence time of run-away CRs in the source proximity is linked to the possibility of accumulating grammage in such regions, which means that a sizable fraction of CR secondaries would be produced in the vicinity of CR sources, contrary to the standard view where secondaries are produced during the time spent by CRs in the Galactic disk. If this is the case, the standard model of Galactic CR propagation should be profoundly revised [see, e.g. 5]. Here we show that, in a partially ionized phases of the interstellar medium (ISM), the ion-neutral and FG dampings can significantly limit the CR self-confinement nearby sources, and for typical ISM conditions, the source grammage is negligible compared to that gained during the CR propagation in the Galaxy. We focus specifically on the warm ionized (WIM) and warm neutral (WNM) phases, which together have a filling factor of $\approx 50 \%$ [6]. A more extensive analysis can be found in [7]. We refer the reader to [8] for the case of a fully ionized medium (filling factor $\approx 50 \%$ [6]), and to [see, e.g. 9] for the case of a cold neutral (CNM) and diffuse molecular (DiM) medium, which exhibit a low filling factor in the ISM ( $\lesssim 1 \%[6]$ ).

\section{Damping of Alfvén waves}

The physical properties of the WIM and WNM are reported in Table 1 (from [10], see also [6]). The ion-neutral damping rate depends on the amount of neutrals and on the chemical species of the colliding particles. In the WNM and WIM the ions are $\mathrm{H}^{+}$, while neutrals are $\mathrm{He}$ (with a $\mathrm{He} / \mathrm{H}$ ratio of $\sim 10 \%$ ) and $\mathrm{H}$ atoms. The transfer of momentum $(\mathrm{mt})$ between ions and neutrals is

Table 1: ISM phases and parameters adopted in this work. $T$ is the gas temperature, $B$ the interstellar magnetic field, $n$ the total gas density, $f$ the ionisation fraction, $\chi$ the helium fraction and $L_{\text {inj }}$ the injection scale of the background magnetic turbulence.

\begin{tabular}{ccccccccc}
\hline & $T(\mathrm{~K})$ & $B(\mu \mathrm{G})$ & $n\left(\mathrm{~cm}^{-3}\right)$ & neutral & ion & $f$ & $\chi$ & $L_{\text {inj }}(\mathrm{pc})$ \\
\hline WIM & 8000 & 5 & 0.35 & $\mathrm{H}, \mathrm{He}$ & $\mathrm{H}^{+}$ & $0.6-0.9$ & $0-0.1$ & 50 \\
\hline WNM & 8000 & 5 & 0.35 & $\mathrm{H}, \mathrm{He}$ & $\mathrm{H}^{+}$ & $7 \times 10^{-3}-5 \times 10^{-2}$ & $0-0.1$ & 50 \\
\hline
\end{tabular}

mainly due to elastic scattering by induced dipole and charge exchange (ce). We refer the reader to [7] for the details, here we report the relevant damping rate, $\Gamma_{d}^{i n}$ in the case of a WIM and a WNM. 



Figure 1: Damping rates $\Gamma_{d}^{i n}$ and $\Gamma_{d}^{\mathrm{FG}}$ (ion-neutral and turbulent) of Alfvén waves in the WNM (left-hand panel) and WIM (right-hand panel) vs. CR energy E. Different colors indicate different values of the hydrogen ionization fraction $f$. Unless specified otherwise, a standard $\chi=0.1 \mathrm{He}$ abundance is assumed. The ISM parameters are summarized in Table 1. In the left-hand panel the dotted lines refers to ion-neutral damping, while the dashed lines to the FG damping. The last are truncated to the minimum energy, $E_{\min }$ (see Sec. 2). The shaded regions represent the range of non-propagation of Alfvén waves (see Sec. 2). In the right-hand panel, the solid lines refer to ion-neutral damping, while the thin dotted lines refer to the case where the contribution to damping from $\mathrm{He}$ is neglected $(\chi=0)$. The dashed lines refer to the FG damping.

Such damping rate is deduced by solving the following equation, for waves of wavenumber $k$,

$$
\omega_{k}^{2}=\frac{2 \Gamma_{d}^{i n}}{v_{i n}-2 \Gamma_{d}^{i n}}\left[(1+\epsilon) v_{i n}-2 \Gamma_{d}^{i n}\right]^{2},
$$

which implies $0<\Gamma_{d}^{i n}<v_{i n} / 2$. Here $m_{n(i)}$ and $n_{n(i)}$ are the mass and density of neutrals (ions), $v_{A, i}=B / \sqrt{4 \pi m_{i} n_{i}}$ is the Alfvén speed in the ions, $B$ is the background magnetic field, $\omega_{k}=k v_{A, i}$. The ion-neutral collision rate, $v_{i n}$, is given by

$$
v_{\text {in }}=\left[\frac{1-f}{2}\left\langle\sigma_{\mathrm{mt}} v\right\rangle_{\mathrm{H}^{+}, \mathrm{H}}+\frac{4 \chi}{5}\left\langle\sigma_{\mathrm{mt}} v\right\rangle_{\mathrm{H}^{+}, \mathrm{He}}\right] \frac{n}{1+\chi},
$$

where $f=\frac{n_{i}}{n_{\mathrm{H}}+n_{i}}$ is the fraction of ionized hydrogen and $\chi=\frac{n_{\mathrm{He}}}{n_{\mathrm{H}}+n_{i}}$ the helium-to-hydrogen ratio. The collisional rate coefficients, $\left\langle\sigma_{\mathrm{mt}} v\right\rangle_{\mathrm{H}^{+}, \mathrm{H}}$ and $\left\langle\sigma_{\mathrm{mt}} v\right\rangle_{\mathrm{H}^{+}, \mathrm{He}}$, are reported by [7]. Alfvén waves are resonantly excited by CR protons at frequency $\omega_{k} \approx v_{\mathrm{A}, i} / r_{\mathrm{L}}$. The effective Alfvén velocity, $v_{\mathrm{A}}$, felt by CRs depends on the coupling between ions and neutrals: at low wavenumber (high CR energy), $\omega_{k} \ll v_{i n}$, ions and neutrals are well coupled and the effective Alfvén speed for CRs is $v_{\mathrm{A}, n}=\frac{B}{\sqrt{4 \pi \mu m_{p} n}}$, where $n$ is the total gas density and $\mu \sim 1.4$ is the mean molecular weight, and $\Gamma_{d}^{i n} \propto E^{-2}$. At high wavenumber (low CR energy), $\omega_{k} \gg v_{i n}$, ions and neutrals are weakly coupled and ion-neutral damping is most effective. The Alfvén speed is the one in the ions, $v_{\mathrm{A}, i}$, and $\Gamma_{d}^{\text {in }} \sim$ const. If $\epsilon<1 / 8$ there is a range of wavenumbers for which waves do not propagate [2]. This is marked as a shaded region in Fig. 1.

In the presence of a pre-existing background turbulence, self-generated Alfvén waves can be damped through turbulent damping (FG). Turbulence may be injected by astrophysical sources at 
scales $L_{\text {inj }}$ much larger than the resonant scale and with turbulent velocity $v_{\text {turb }}$. For waves resonant with particles of energy $E$ the damping rate is given by $[3,11]$

$$
\Gamma_{d}^{\mathrm{FG}}=\left(\frac{v_{\mathrm{turb}}^{3} / L_{\mathrm{inj}}}{r_{\mathrm{L}} v_{\mathrm{A}}}\right)^{1 / 2}
$$

where $v_{\mathrm{A}}$ is the effective Alfvén speed felt by CRs. We assume $v_{\text {turb }}=v_{\mathrm{A}, n}$ at the injection scale (at large scales ions and neutrals are well coupled). The FG damping rate is shown in Fig. 1 for the WIM and WNM. For large enough neutral density, as in the WNM, the turbulence responsible for the FG damping can be damped by ion-neutral friction at a scale, $l_{\min }=1 / k_{\min }$ (see [9] and references therein). This gives a minimum particle energy, $E_{\min }$, such that $r_{\mathrm{L}}\left(E_{\min }\right)=l_{\min }$, below which the FG damping cannot suppress the self-generated waves [9]:

$$
\frac{1}{l_{\min }}=L_{\mathrm{inj}}^{1 / 2}\left(\frac{2 \epsilon v_{i n}}{v_{\mathrm{A}, n}}\right)^{3 / 2} \sqrt{1+\frac{v_{\mathrm{A}, n}}{2 \epsilon v_{i n} L_{\mathrm{inj}}}} .
$$

In Fig. 1 the FG damping rate for the WNM is truncated at $E_{\min }$ while in the WIM the wave cascade is always faster than ion-neutral damping and there is no $E_{\min }$.

The non-linear Landau (NLL) damping is due to the interaction between the background thermal ions and the beat of two Alfvén waves. The damping rate is given by [4]

$$
\Gamma_{d}^{\mathrm{NLL}}=\frac{1}{2} \sqrt{\frac{\pi}{2}\left(\frac{k_{\mathrm{B}} T}{m_{\mathrm{p}}}\right)} \frac{I\left(k_{\mathrm{res}}\right)}{r_{\mathrm{L}}},
$$

where $k_{\mathrm{B}}$ is the Boltzmann constant, $T$ the gas temperature and $I\left(k_{\mathrm{res}}\right)$ is the wave energy density (see Sec. 3 below for the definition) at the resonant wavenumber $k_{\text {res }}=1 / r_{\mathrm{L}}$.

\section{Cosmic ray transport in the vicinity of SNRs}

We consider the propagation of runaway CRs in the vicinity of SNRs, in a region embedded in a turbulent magnetic field, with a ordered component of strength $B_{0}$. We consider the CR scattering on self-generated Alfvén waves of wavenumber $k$ and amplitude $\delta B / B_{0}$ and that propagate solely along the uniform background field $B_{0}$. In the limit of $\delta B / B_{0} \ll 1$, the CR diffusion along field lines can be treated in the quasi-linear regime, with a diffusion coefficient [12] $D(E)=\left.\frac{4 \pi c r_{\mathrm{L}}(E)}{3 I\left(k_{\mathrm{res}}\right)}\right|_{k_{\mathrm{res}}=1 / r_{\mathrm{L}}}=$ $\frac{D_{\mathrm{B}}(E)}{I\left(k_{\mathrm{res}}\right)}$, where $c$ is the speed of light, $I\left(k_{\mathrm{res}}\right)=\delta B\left(k_{\mathrm{res}}\right)^{2} / B_{0}^{2}$ is the wave energy density calculated at the resonant wavenumber $k_{\text {res }}=1 / r_{\mathrm{L}}$, and $D_{\mathrm{B}}(E)=(4 \pi / 3) c r_{\mathrm{L}}(E)$ is the Bohm diffusion coefficient. We also assume a flux-tube approximation (see [7] and references therein), where we neglect the diffusion across field lines, which is suppressed in the $\delta B / B_{0} \ll 1$ regime (see e.g. [13]). This approach is roughly valid for distance from the source below the coherence length, $L_{\mathrm{c}}$, of the background magnetic turbulence. At distances larger than $L_{\mathrm{c}}$, diffusion becomes 3-D and the CR density drops quickly. In the Galactic disk, $L_{\mathrm{c}}$ is estimated from observations and may range from few pc to $\approx 100 \mathrm{pc}$, depending on the ISM phase [see, e.g., 14, and references therein]. The 
coupled CR and wave transport equations read $[8,15]$

$$
\begin{aligned}
& \frac{\partial P_{\mathrm{CR}}}{\partial t}+v_{\mathrm{A}} \frac{\partial P_{\mathrm{CR}}}{\partial z}=\frac{\partial}{\partial z}\left(\frac{D_{\mathrm{B}}}{I} \frac{\partial P_{\mathrm{CR}}}{\partial z}\right) \\
& \frac{\partial I}{\partial t}+v_{\mathrm{A}} \frac{\partial I}{\partial z}=2\left(\Gamma_{\mathrm{CR}}-\Gamma_{d}\right) I+Q .
\end{aligned}
$$

Here $v_{\mathrm{A}}$ is an effective Alfvén velocity, while $P_{\mathrm{CR}}$ is the $\mathrm{CR}$ partial pressure normalized to the magnetic field pressure $P_{\mathrm{CR}}=\frac{4 \pi}{3} c p^{4} \frac{f(p)}{B_{0}^{2} / 8 \pi}$ and $p$ is the particle momentum. The term $2 \Gamma_{\mathrm{CR}} I=$ $-v_{\mathrm{A}} \frac{\partial P_{\mathrm{CR}}}{\partial z}$ represents the growth of waves due to the streaming instability, and $\Gamma_{d}$ includes the wave damping rates illustrated in Sec. 2. $Q=2 \Gamma_{\mathrm{d}} I_{0}$ includes the possible injection of turbulence from an external source, where $I_{0}$ is such that typical values of the Galactic CR diffusion coefficient are recovered at large distances from the source [see, e.g., 16]. The coordinate $z$ is taken along $B_{0}$ and $z=0$ refers to the center of the CR source and the equations are solved numerically with the initial conditions: $P_{\mathrm{CR}}=P_{\mathrm{CR}}^{0}$ if $z<R_{\mathrm{esc}}(E), P_{\mathrm{CR}}=P_{\mathrm{CR}}^{0}=0$ if $z>R_{\mathrm{esc}}(E)$, and $I=I_{0}$ everywhere. $P_{\mathrm{CR}}^{0}$ is the initial CR pressure inside a region of size $R_{\mathrm{esc}}(E)$, namely the escape radius. The latter is determined as described in $[8,15]$. We impose a symmetric CR distribution at $z=0$, and the boundary conditions: $P_{\mathrm{CR}}=0, I=I_{0}$ at $z \gtrsim L_{\mathrm{c}}$. The specific location of the free escape boundary does not affect appreciably the results.

Throughout our calculations we assume: (i) a total CR energy $E_{\mathrm{CR}}=10^{50} \mathrm{erg}$, a $E^{-2.0}$ power-law spectrum between $1 \mathrm{GeV}-5 \mathrm{PeV}$; (ii) the typical ISM diffusion coefficient is $D(E)=$ $D_{0}(E / 10 \mathrm{GeV})^{0.5}$ with $D_{0}=10^{28} \mathrm{~cm}^{2} \mathrm{~s}^{-1}$ [see, e.g., 16].

\section{Results}

In order to determine the moment of escape of CRs of energy $E$ from the remnant, we define the half-time, $t_{1 / 2}(E, R)$ of a CR cloud as the time after which half of the particles initially confined within a region of size $R$ escaped that region (see $[8,15]$ ). The temporal and spatial evolution of such cloud is governed by the equations and the initial/boundary conditions illustrated in Sec. 3 .

The escape time, $t_{\mathrm{esc}}(E)$ is estimated as the time such that the half-time of a CR cloud of radius $R_{\mathrm{SNR}}, t_{1 / 2}\left(E, R_{\mathrm{SNR}}\right)$, equals the age of a remnant of radius $R_{\mathrm{SNR}}[8,15]$. The temporal evolution of the remnant radius is described in detail by [7] and takes into account the different phases of evolution: free expansion, adiabatic and rediative, which depend on the supernova explosion energy, on the ejecta mass, an on the background gas density. We also assume that at the beginning of the radiative phase the CR acceleration becomes ineffective and that all CRs are instantly released.

Fig. 2 shows as a function of the particle energy the radius and age of the remnant at the moment of escape. Qualitative, one can see that particles with higher energy escape earlier than the low energy particles (see, e.g., [17] and [8, 15]). Notice that in a WIM, $R_{\text {esc }}$ and $t_{\text {esc }}$ tend to decrease with a smaller ionization fraction. The effect of neutrals is well visible in the energy range $\sim 1-10 \mathrm{TeV}$, where the ion-neutral damping rate decreases from a roughly constant value at different particle energies, depending on the value of $f$ and $\chi$ (see Tab. 1), as shown in Fig. 1. The decrease of $\Gamma_{d}^{i n}$ corresponds to an increase of $t_{\mathrm{esc}}$, namely to a better CR confinement. In the case of a WNM, instead, particles appear slightly better confined for a smaller value of $f$. This can be explained by the fact that, while the $\Gamma_{d}^{\text {in }}$ is practically unchanged below $\sim 10 \mathrm{TeV}$ for $f \sim 7 \times 10^{-3}-5 \times 10^{-2}$, 
WNM
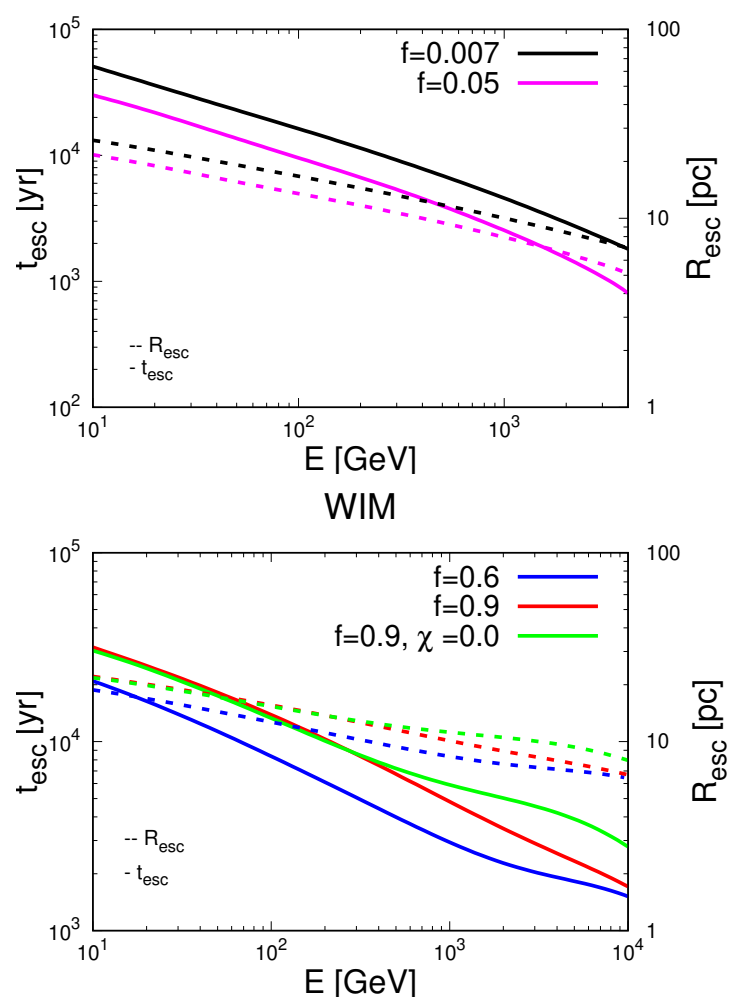

WNM
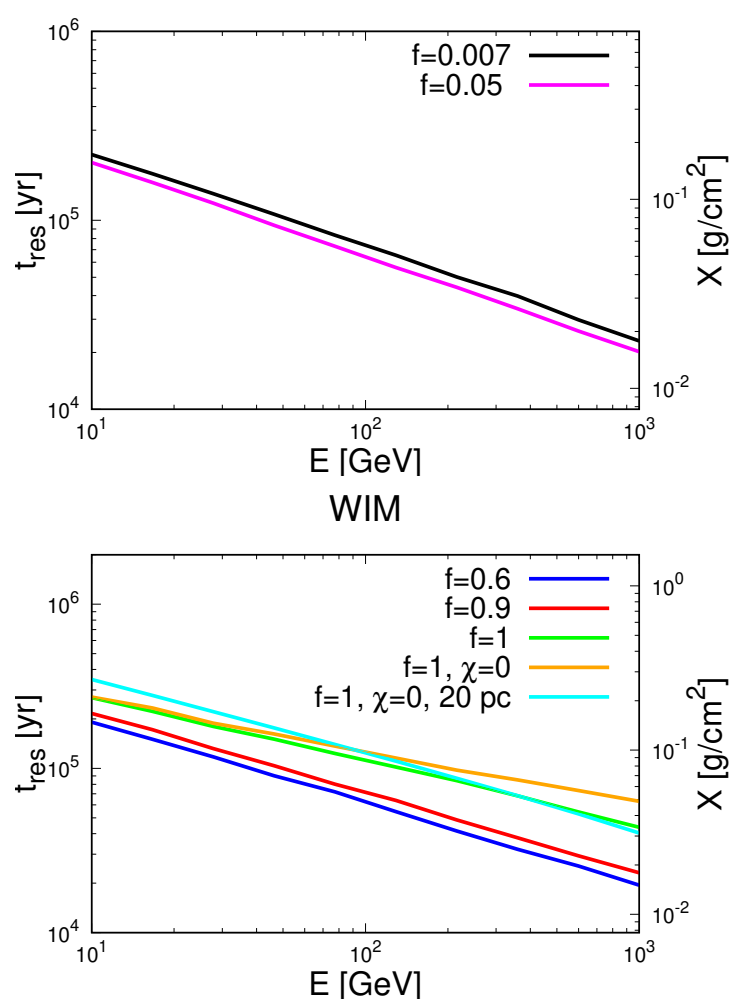

Figure 2: Case of WNM and WIM as marked. Left panels: SNR age and radius at the time of escape of CRs, as a function of the CR energy. Right panels: Residence time of CRs in a region of $\sim 100 \mathrm{pc}$ around the source, as a function of the CR energy, and the corresponding grammage. Different colors are used for different values of the hydrogen ionization fraction $f$, while the helium-to-hydrogen ratio $\chi=0.1$ (see Table 1.) The cyan curve in the WIM case is computed by assuming that particles of all energies escape at SNR radius of $20 \mathrm{pc}$, as assumed by [5].

the effective Alfvén speed which at these energies is roughly that of ions ( see Sec. 2), is a factor of $\sim 2-3$ larger for $f=7 \times 10^{-3}$. This reflects on a decrease by the square root of the same amount of the FG damping rate, as shown in Fig. 1, but also in an increase of the growth rate term, which is proportional to $v_{\mathrm{A}}$. The overall effect is a slightly better confinement at smaller $f$ as a result of an enhanced wave growth rate and reduce FG damping. In the case of a WIM $v_{\mathrm{A}} \approx v_{A, n}$ at all energies and the dominant effect when decreasing $f$ is the enhancement of ion-neutral damping.

A formal calculation of the grammage should be performed by solving the transport equation for nuclei including spallation [see, e.g., 12]. Here we adopt the following approach: we consider $N_{0}$ particles of energy $E$ instantly released by a source in a region of constant density $\rho=\mu m_{p} n$ and free escaping at a boundary located at $L_{\mathrm{c}}$ from the source. At any time $t, N_{\text {in }}(t)$ particles are still in the region and $N_{\mathrm{esc}}=N_{0}-N_{\text {in }}(t)$ have escaped and gained the grammage $X(t)=\rho c t$. The average grammage accumulated by all escaped particles is given by

$$
\langle X\rangle=\frac{1}{N_{0}} \int_{0}^{\infty} \rho c t \frac{d N_{\mathrm{esc}}}{d t} d t=\frac{\rho c}{N_{0}}\left[\int_{0}^{\infty} N_{\mathrm{in}}(t) d t-\lim _{t \longrightarrow \infty}\left(t N_{\mathrm{in}}(t)\right)\right]=\rho c \tau_{\mathrm{res}},
$$

where in the last equality is also defined the residence time $\tau_{\text {res. }} N_{\text {in }}(t)$ can be calculated from the 
CR pressure (see Sec. 3): $N_{\text {in }}(t)=\frac{N_{0}}{R_{\mathrm{esc}} P_{\mathrm{CR}}^{0}} \int_{0}^{L_{\mathrm{c}}} P_{\mathrm{CR}}(z, t, E) d z$, where we considered the CR cloud size $R_{\text {esc }}(E)$ at the moment of injection. Fig. 2 shows the residence time of CRs in a region of $\sim 100 \mathrm{pc}$ around the remnant, and the related grammage, as a function of $f$ and $\chi$ and for a WNM and a WIM. The found grammage is nearly two orders of magnitudes smaller than that accumulated in the disk (see e.g. [18]). This is true also in the unlikely case of a fully ionized WIM. Any addition of neutral gas, even only $10 \%$ of neutral Helium, further reduces the residence time. This is at odds with what was suggested by [5], which finds a grammage nearly a factor of ten larger. This is explained by the fact that: i) they neglect the FG damping and the contribution of neutral Helium to ion-neutral damping; ii) assume a $20 \%$ acceleration efficiency, that contribute to enhance the streaming instability (while we assume 10\%); iii) impose that all particles escape at a SNR radius of $20 \mathrm{pc}$, which is smaller than our release radius at low energies, that also enhances the steaming instability; iv) assume a slightly larger total gas density.

\section{Conclusions}

We investigated the escape of CRs from a SNR embedded in a WNM and a WIM, we studied the residence time in the source region and the accumulated grammage. We consider the selfconfinement by CR streaming instability and the relevant damping processes, namely turbulent, ionneutral and non-linear Landau damping. For the ion-neutral damping we used up-to-date damping coefficients, based on an accurate experimental or theoretical determination of the relevant cross sections. If such grammage was a sizable fraction of that inferred from observations, the standard picture of CR Galactic propagation, where secondaries are produced during the whole time spent by CRs in the disk, should be substantially revised.

We conclude that, in the considered scenario, under the conditions typically found in the WIM and WNM of the Galactic disk, the grammge accumulated by CRs within $\sim 100 \mathrm{pc}$ from the source is negligible. A similar result was found in the case of a HIM by [8], where the residence time tends to be larger than in a partially neutral medium, but the total ISM is at least a factor of ten smaller. This makes less appealing the possibility of an important fraction of secondary production in the source region.

\section{References}

[1] R. Kulsrud and W.P. Pearce, The Effect of Wave-Particle Interactions on the Propagation of Cosmic Rays, ApJ 156 (1969) 445.

[2] E.G. Zweibel and J.M. Shull, Confinement of cosmic rays in molecular clouds, ApJ 259 (1982) 859.

[3] A.J. Farmer and P. Goldreich, Wave Damping by Magnetohydrodynamic Turbulence and Its Effect on Cosmic-Ray Propagation in the Interstellar Medium, ApJ 604 (2004) 671 [astro-ph/0311400].

[4] J. Wiener, E.G. Zweibel and S.P. Oh, Cosmic Ray Heating of the Warm Ionized Medium, ApJ 767 (2013) 87 [1301.4445]. 
[5] M. D'Angelo, P. Blasi and E. Amato, Grammage of cosmic rays around Galactic supernova remnants, Phys. Rev. D 94 (2016) 083003 [1512.05000].

[6] K.M. Ferrière, The interstellar environment of our galaxy, Rev. Mod. Phys. 73 (2001) 1031 [astro-ph/0106359].

[7] S. Recchia, D. Galli, L. Nava, M. Padovani, S. Gabici, A. Marcowith et al., Grammage of cosmic rays in the proximity of supernova remnants embedded in a partially ionized medium, arXiv e-prints (2021) arXiv:2106.04948 [2106. 04948].

[8] L. Nava, S. Recchia, S. Gabici, A. Marcowith, L. Brahimi and V. Ptuskin, Non-linear diffusion of cosmic rays escaping from supernova remnants - II. Hot ionized media, MNRAS 484 (2019) 2684 [1903. 03193].

[9] L. Brahimi, A. Marcowith and V.S. Ptuskin, Nonlinear diffusion of cosmic rays escaping from supernova remnants: Cold partially neutral atomic and molecular phases, A\&A 633 (2020) A72 [1909.04530].

[10] P. Jean, W. Gillard, A. Marcowith and K. Ferrière, Positron transport in the interstellar medium, A\&A 508 (2009) 1099 [0909.4022].

[11] H. Yan and A. Lazarian, Cosmic-Ray Scattering and Streaming in Compressible Magnetohydrodynamic Turbulence, ApJ 614 (2004) 757 [astro-ph/0408172].

[12] V.S. Berezinskii, S.V. Bulanov, V.A. Dogiel and V.S. Ptuskin, Astrophysics of Cosmic Rays (1990).

[13] F. Casse, M. Lemoine and G. Pelletier, Transport of cosmic rays in chaotic magnetic fields, Phys. Rev. D 65 (2002) 023002 [astro-ph/0109223].

[14] L. Nava and S. Gabici, Anisotropic cosmic ray diffusion and gamma-ray production close to supernova remnants, with an application to W28, MNRAS 429 (2013) 1643 [1211.1668].

[15] L. Nava, S. Gabici, A. Marcowith, G. Morlino and V.S. Ptuskin, Non-linear diffusion of cosmic rays escaping from supernova remnants - I. The effect of neutrals, MNRAS 461 (2016) 3552 [1606 .06902].

[16] A.W. Strong, I.V. Moskalenko and V.S. Ptuskin, Cosmic-ray propagation and interactions in the galaxy, Ann. Rev. Nucl. Part. Sci. 57 (2007) 285-327.

[17] S. Gabici, Cosmic ray escape from supernova remnants, Mem. Soc. Astron. Italiana 82 (2011) 760 [1108.4844].

[18] S. Gabici, C. Evoli, D. Gaggero, P. Lipari, P. Mertsch, E. Orlando et al., The origin of Galactic cosmic rays: Challenges to the standard paradigm, Int. J. Mod. Phys. D 28 (2019) 1930022 [1903.11584]. 